\title{
Computational Study of Aerodynamics of Low Aspect Ratio Wings
}

\author{
J. Liu1 ${ }^{1 *}$, P. F. Weng ${ }^{2}$
}

Shanghai University, Shanghai Institute of Applied Mathematics and Mechanics, Shanghai, 200072, China

${ }^{1}$ Email: circumvent@163.com

2 Email: pfweng@staff.shu.edu.cn

\begin{abstract}
Micro air vehicle (MAV) has been always at the frontier of the current aeronautical research. However, classical aerodynamic theory, which can accurate predict large-scale aircrafts, is generally not applicable for low aspect ratio (LAR) wings at low Reynolds number. Few investigations have been done for thin, cambered LAR wings [1, 2] commonly used on MAVs, yet most of them focused on experimental attempts and theoretically solutions. In present study, LAR wing with Zimmerman planform, which was proven to be more desirable in MAV design[3] at moderate angles of attack, is studied numerically to investigate effects of AR and camber on the performance of LAR wing.

Wing models in the present study have thickness-to-chord ratio of $2 \%$, round leading edges and sharp trailing edges. Reynolds number is set to $1.4 \times 10^{5}$ for all computational cases considering the general MAV cruise speed of $15 \mathrm{~m} / \mathrm{s}$. Steady-state incompressible Navier-Stokes equations in three-dimensions are solved using the artificial compressibility method[4]. C-H grid, with dimensions of $123 \times 50 \times 31$, is generated using hyperbolic grid generation method. The accuracy of the flow computations is validated by the flow over a thin wing[1], which demonstrates that the code is accurate and robust for aerodynamic analysis of MAV.

Firstly, flows over a series of wings with distinct Semi-AR, ranging from 0.5 to 2.0 with the interval of 0.5 , are studied. Results show that AR is a key design parameter in MAV aerodynamic design. It is more beneficial to have a wing with an AR as high as possible while still within the design size. Secondly, cambers of $2 \%, 4 \%$ and $6 \%$ are considered compared with the wing with no camber. It concludes that for the wing with no camber there exits a big reverse flow region over the majority of the leading-edge on the upper surface. While cambered wings have two distinct reverse flow regions: near the leading edge and near the trailing edge. The bigger the camber is, the smaller the leadingedge reverse flow region is. While the bigger the camber is, the bigger the trailing edge flow region is. Cambered wings lead to better aerodynamic characteristics because of an increase in lift, even thought drag also increases.
\end{abstract}

\section{REFERENCES}

1. Pelletier A, Mueller T J. Low Reynolds number aerodynamics of low-aspect-ratio, thin/flat/camberedplate wings. Journal of Aircraft, 2000; 37(5): $825-832$

2. Torres E T. Acrodynamics of low aspect ratio wings at low reynolds numbers with applications to micro air vehicle design and optimization: [dissertation], Univ. of Notre Dame, Indiana, 2002

3. Liu J, Wong P. A numerical investigation of aerodynamics of micro air vehicle. In: Proceeding of the Fifth International Conference on Fluid Mechanics, 2007, Shanghai, China. In Press, Accepted Manuscript

4. Rogers S E, Kwak D. Steady and unsteady solutions of the incompressible Navier-Stokes equations. AIAA Journal, 1989; 29(4): 603-610 